\title{
Necessity of Evaluation of Non-coding RNAs in Clinical Trial for Target Therapy
}

\author{
Seyed Hassan Saadat \\ Personalized Medicine Research Center, Tehran, Iran \\ Email: hsaadat@cikd.ca
}

Genomic and proteomic approaches have improved the understanding of the mechanisms involved in cancer. Genomic mutations in cancer indicate that many mutations or altered copy numbers in cancer often occur in non-coding regions of the DNA including microRNAs (miRNAs), long noncoding RNAs (lncRNAs), small interfering RNAs (siRNAs), and antisense RNAs (Li et al, 2017; Yoon \& Rossi, 2018; Yu, Jian, Allan, \& Tu, 2019).

Noncoding RNAs, including lncRNAs and miRNAs, are potential therapeutic targets because they have the potential to be applied in the diagnosis and prognosis of a number of cancers. Furthermore, their regulations are implicated in development and progression of many kinds of malignancies e.g., proliferation, invasion, metastasis, angiogenesis and drug resistance, thus suggesting its potential in targeted therapy (Smolle, Calin, Pichler, \& Calin, 2017; Vo et al., 2019; Zhang \& Xin, 2018). Therefore, RNA profiling using bioinformatics tools and the development of databases can be helpful in development of targeted research in this regard, where in-depth understanding of their multiple mechanisms will be helpful in clarifying their substantial role in regulation of many genes involved in cancer. For instance, anti-miRNA oligonucleotides (AMO) are considered to be capable of suppressing the function of oncomirs, leading to inhibition of tumor growth. Clinical trials and increased success rates in noncoding RNAs therapy are considered to be an opportunity for cancer treatment.

\section{References}

Li, Y., Xu, Q., Lv, N., Wang, L., Zhao, H., Wang, X., ... \& Yu, L. (2017). Clinical implications of genome-wide DNA methylation studies in acute myeloid leukemia. Journal of Hematology and Oncology, 10(1), 41.

Smolle, M. A., Calin, H. N., Pichler, M., \& Calin, G. A. (2017). Noncoding RNAs and immune checkpoints—clinical implications as cancer therapeutics. The FEBS journal, 284(13), 1952-1966.

Vo, J. N., Cieslik, M., Zhang, Y., Shukla, S., Xiao, L., Zhang, Y., ... \& Robinson, D. R. (2019). The landscape of circular RNA in cancer. Cell, 176(4), 869-881.

Yoon, S., \& Rossi, J. J. (2018). Therapeutic potential of small activating RNAs (saRNAs) in Human Cancers. Current Pharmaceutical Biotechnology, 19(8), 604-610.

Yu, A. M., Jian, C., Allan, H. Y., \& Tu, M. J. (2019). RNA therapy: Are we using the right molecules? Pharmacology and Therapeutics, 196, 91-104.

Zhang, M., \& Xin, Y. (2018). Circular RNAs: a new frontier for cancer diagnosis and therapy. Journal of Hematology and Oncology, 11(1), 21. 